\title{
Geschlechtergerechte Paartherapie mit heterosexuellen Paaren: Reflexionen zu einem utopischen Unterfangen
}

\author{
Sabine Kirschenhofer
}

Online publiziert: 10. Mai 2019

(C) Der/die Autor(en) 2019

\begin{abstract}
Zusammenfassung In diesem Text möchte ich $\mathrm{zu}$ einer Auseinandersetzung mit Geschlecht und Geschlechterverhältnissen einladen - als systemische Therapeutin wähle ich dafür das mir vertraute Arbeitsfeld der Paartherapie mit heterosexuellen Paaren. Nach einer Begriffsklärung, was geschlechtergerecht im Kontext von Paartherapie für mich bedeutet, werde ich aus einer feministischen Perspektive mit einem kritischen Blick auf zeitgenössische patriarchale Verhältnisse operieren: Ich werde dies zuerst anhand eines verdichteten Fallbeispiels tun; danach prüfe ich die systemische Therapie darauf, wie sie für dieses Thema nutzbar gemacht werden kann und wo sie versagt. Im letzten Abschnitt biete ich Empfehlungen zur Selbstreflexion an, um sich im Balanceakt eines Sowohl als auch zu üben - sowohl der Berücksichtigung von Unterschieden als auch einem Offenbleiben für die Einzigartigkeit von Menschen jenseits von Geschlecht.
\end{abstract}

Schlüsselwörter Paartherapie · Systemisch · Heterosexuell · Geschlecht · Patriarchat · Feminismus

\section{Gender-just couples' therapy with heterosexual couples: Reflections on an utopian project}

Summary In this article I wish to invite to a serious reflection on gender relations, and as a systemic therapist I choose a familiar area of work, namely couples' therapy with heterosexual couples. At the beginning I will offer a clarification of what "gender-just" means for me in the context of couples' therapy; then I will use a feminist perspective with a critical gaze on pa-

\footnotetext{
S. Kirschenhofer $(\bowtie)$

Institut für Paar- und Familientherapie, Wiener

Sozialdienste, Praterstraße 40/10, 1020 Wien, Österreich

sabine.kirschenhofer@wiso.or.at
}

triarchal relations nowadays: first I will demonstrate this with a condensed case example; then I wish to examine the framework of systemic therapy with regard to its potential as well as its failings. In the last section I offer reflexive ideas and recommendations for approaching the balancing act of "both as well as"-a consideration of difference as well as remaining open to the uniqueness of people irrespective of gender.

Keywords Couples' therapy · Systemic · Heterosexual · Gender · Patriarchy $\cdot$ Feminism

Was kann geschlechtergerecht im Kontext von Paartherapie bedeuten?

Geschlecht als zentrale Strukturkategorie unserer Gesellschaft betrifft auch den therapeutischen Raum, den ich mit Rachel Hare-Mustin (1994) als verspiegelten Raum im Sinn eines Spiegel(n)s dominanter Diskurse sehe. Ich interessiere mich dafür, wie man in der paartherapeutischen Arbeit allen Geschlechtern gerecht werden kann in einem gesellschaftlichen Kontext, der von Ungleichheit und Ungerechtigkeit bestimmt wird. Wiewohl wir darauf nicht direkt in paartherapeutischen Gesprächen einwirken können, so bedarf es einer Vergegenwärtigung patriarchaler Strukturen, die ökonomische, soziale und emotionale Folgen zeitigen, die so gut wie jedes psychotherapeutische Gespräch durchdringen. Zusätzlich leben wir aktuell in einer Zeit polarisierter Diskurse: Frauenvolksbegehren 2.0 versus rechtskonservativem Backlash, inklusive der perfiden Strategie, feministische Argumente $\mathrm{zu}$ missbrauchen, um rassistische Hetze zu betreiben. Die Projektion des Patriarchats und der Unterdrückung der Frau auf Migrant*innen bzw. Muslim*innen ist eine Ablenkungsstrategie, die dominante Diskurse noch weiter nach rechts verschiebt. 
Wenn wir soeben Ausgeführtes ernst nehmen, so bedeutet das, dass Geschlechtergerechtigkeit und das Anstreben solcher in der Paartherapie teilweise Utopie bleiben muss. Trotzdem als Therapeutin danach $\mathrm{zu}$ handeln, geschlechtergerecht im Sinn von Menschen gerecht $\mathrm{zu}$ werden in ihrer Unterschiedlichkeit und Einzigartigkeit, ist für mich die Bedeutung des Begriffs, die ich für eine wesentliche Aufgabe in Paartherapien halte. So komplex erscheint mir diese Aufgabe auch deshalb, weil die*der Therapeut*in selbst in seiner*ihrer geschlechtlichen Performanz, mit seiner*ihrer geschlechtlichen Sozialisierung und Biografie, Wahrnehmungen und Ideen von Weiblichkeit, Männlichkeit und Paarbeziehungen mächtige*r Akteur*in im paartherapeutischen Geschehen ist.

Warum steht im Titel „heterosexuell“? Weil es mir darum geht, die Norm sichtbar zu machen ${ }^{1}$. Der Großteil der Fachliteratur und Fortbildungsangebote tragen Paartherapie im Titel - es geht aber fast immer um heterosexuelle Paare, was nicht ausgewiesen wird, denn schließlich handelt es sich um die Mehrheit und die Norm, die Dominanz der heterosexuellen Matrix (Butler 1991). Die Normalität bleibt unsichtbar und wenn die Normalität u.a. eine heteronormative, patriarchale und rassistische ist, dann bedarf es analytischer und reflexiver Arbeit; für mich bedeutet das eine Auseinandersetzung mit herrschenden gesellschaftlichen Verhältnissen und den eigenen Verstrickungen in diese sowie ein kritisches Hinterfragen der Auswirkungen der eigenen Privilegien ${ }^{2}$ : Ich wage zu behaupten, dass nur wenige Kolleginnen und Kollegen dazu bereit sind - zu verlockend ist das Selbstbild, progressiv, tolerant, gendersensibel, antirassistisch ${ }^{3}$ usw. zu sein. Im Kontext heterosexueller Paartherapie führt dies zu einem Verfestigen von leidgenerierenden Strukturen und Problemen, die die Betroffenen in die Therapie geführt haben.

\section{Und täglich grüßt das Patriarchat - „der Mann als drittes Kind"،}

Perspektivisch hilfreich und nützlich finde ich nach wie vor den in die Jahre gekommenen Begriff des Patriarchats - in Anlehnung an die Historikerin Gerda Lerner (1986) definiert als Manifestation und Institutionalisierung männlicher Dominanz in Familie und

\footnotetext{
1 „Naming the norm“ ist eine Strategie antirassistischer, postkolonialer und feministischer Bewegungen, die es ermöglicht, Normales, Natürliches, Selbstverständliches durch Benennung der normativen Unsichtbarkeit zu entreißen: So werden diskursive Qualitäten von Konstruktion und Macht ausgewiesen und veränderbar.

2 Der Text Deconstructing heterosexism von Christi McGeorge und Thomas Stone Carlson (2011) ist zum Beispiel lehrreich für heterosexuelle Paar- und Familientherapeut*innen, die sich mit ihren heteronormativen Annahmen und heterosexuellen Privilegien auseinandersetzen möchten.

3 Tupoka Ogette (2017) nennt diese behagliche Selbstverortung Weißseins „Happyland“.
}

Gesellschaft. Weiters bediene ich mich des Begriffs der hegemonialen Männlichkeit, einer Leitkategorie in den men's studies: Michael Meuser (2009, S. 161) schlägt vor, hegemoniale Männlichkeit als generatives Prinzip der Konstruktion von Männlichkeit zu begreifen und er plädiert dafür, Verhältnisse von Macht und Herrschaft, von Dominanz und Unterordnung in der hetero- wie in der homosozialen Dimension zu betrachten ${ }^{4}$. Wiewohl gerade der Feminismus der Dekonstruktion (vgl. Butler 1991) und auch die weitreichende formale Gleichstellung der Geschlechter in Europa den Eindruck erwecken, wir wären bereits im Zeitalter des Postfeminismus angelangt, so kann von einer ungebrochenen Wirkmacht patriarchaler Strukturen ausgegangen werden. Um zu veranschaulichen, wie sich meines Erachtens Strukturelles in der Intimsphäre von heterosexuellen Paarbeziehungen zeigt, werde ich eine verdichtete Fallvignette zeigen - sie beinhaltet eine Konstellation, die mir im Laufe der Jahre im Institut für Paar- und Familientherapie immer wieder begegnet ist. Typischerweise fällt bereits bei der telefonischen Anmeldung (mehrheitlich durch die Frau) der Satz: „Mein Mann ist das dritte Kind“.

Nennen wir sie Peter und Petra Meier, ein Paar Ende dreißig mit zwei Kindern im Alter von 8 und 5, beide haben Matura, er arbeitet Vollzeit, sie Teilzeit, ein Arrangement seit ihrer Kinderkarenz; seit 12 Jahren befinden sie sich in einer Lebensgemeinschaft. Petra hat die Paartherapie initiiert, weil sie seit langem sehr unzufrieden in der Paarbeziehung ist.

Petra ist seit der Geburt des ersten Kindes hauptsächlich zuständig für den Haushalt, die Kinderbetreuung und -erziehung sowie für das Management aller familiären Angelegenheiten ... „das hat sich so ergeben“. Petra fühlt sich immer wieder erschöpft und frustriert, weil ihr alles $\mathrm{zu}$ viel ist und sie sich von Peter alleine gelassen fühlt, sie erlebt ihn mit seinen Bedürfnissen und seiner Passivität bezüglich aller Haushalts- und Familienangelegenheiten als Belastung. Sie möchte mehr Unterstützung von Peter, mehr Gespräch, mehr Verbindendes als das alltägliche Funktionieren der Familie. Sie hat immer wieder auch ein schlechtes Gewissen, weil sie zu müde ist für Sex, den er einfordert. Wenn sie mit ihm reden möchte, um etwas $\mathrm{zu}$ verändern, erlebt sie ihn als abwertend und distanziert - er zieht sich beleidigt zurück.

Peter ist ihr zuliebe mitgekommen in die Paartherapie, ihn stört vor allem ihre chronische Unzufriedenheit, die ihrem Perfektionismus geschuldet ist - sie will, dass er zum Erfüllungsgehilfen ihrer Ansprüche an Haushalt und Kinder-

\footnotetext{
${ }^{4}$ Heterosozial im Sinn der Hauptachse männlicher Macht im Verhältnis von Mann und Frau; homosozial meint männliche Herrschaft in der Beziehung von Männern untereinander.
} 
erziehung werde; wenn er sie unterstützt, mache er es aber sowieso falsch, also kann er es gleich lassen. Er fühlt sich von ihr zu wenig geschätzt in dem, was er leistet - schließlich verdient er den Großteil des Geldes, der ihren Lebensstil finanziert mit Urlauben und Skifahren usw. Er ist oft erschöpft, fühlt sich von ihr bedrängt, wenn sie reden will. Dass es schon lange fast keine körperliche Nähe mehr gibt, stört ihn sehr - wenn es zu Sex kommt, dann geht es von ihm aus, aber er weiß gar nicht, ob sie wirklich Lust hat.

In dieser sich im Erstgespräch zeigenden Variante zeitgenössischen Paar-Leidens zeigt sich unter anderem Folgendes:

- Ein Nebeneinander und eine Gleichzeitigkeit an traditionellen und modernen Geschlechter-RollenPrägungen durchkreuzen die Erwartungen an sich selbst und den*die andere*n - eine möglicherweise als partnerschaftlich wahrgenommene Paarbeziehung vor den Kindern wird (im Zuge der so typischen Retraditionalisierung der Geschlechterrollen nach der Geburt des ersten Kindes mit der Karenz der Frau) überlagert von einer traditionelleren Form, die dem davor Gelebten und möglicherweise auch Bevorzugten widerspricht. Hier sehen wir oft auch widersprüchliche Wünsche von Frauen, die Partnerschaftlichkeit einfordern, bei Kindererziehung oder Haushaltsführung jedoch eine maßregelnde und überlegene Position einnehmen.

- Im diskursiven Verhandeln von Wünschen und Bedürfnissen sind wir mit dem Problem konfrontiert, dass hegemoniale Männlichkeit bzw. patriarchale Privilegien für Männer eine Hierarchisierung von Bedürfnissen und Wünschen impliziert. Die eigenen (männlichen) Erfahrungen brauchen weder einer Analyse oder Verantwortung zugeführt werden, sie stellen die Norm(alität) dar (Dickerson 2013). Das Sich-Erklären-Wollen und Verstandenwerden-Wollen ist ein Bedürfnis der schwächeren Seite und geht öfter von weiblicher Seite aus, stößt aber auf taube Ohren, wenn der Partner sich nicht von Anspruchshaltungen und Orientierungen an hegemonialer Männlichkeit lösen will. Vor dieser kulturellen Folie ist es auch verständlich, dass manche Männer das viele Reden über die Beziehung abwerten als unproduktiv, sinnlos, nervig: Wenn männliche Identität mit Wissen, Kompetenz und Rechthaben verknüpft ist, dann irritieren davon abweichende Erfahrungen und partnerschaftliche Gespräche, weil ein Verlust von Vertrautem (und das inkludiert Privilegien) riskiert werden könnte.

- Ein Festhalten an patriarchalen Privilegien bedeutet, dass es für manche Männer so unerträglich ist, mit Kritik konfrontiert zu werden: Sie hören nicht, dass ein bestimmtes Verhalten die Partnerin stört, sondern fühlen sich als gesamte Person kritisiert. Diese Generalisierungstendenz von Kritik (vom Verhalten weg zur ganzen Person) zeigt sich meiner Erfahrung nach mehrheitlich bei Männern und verweist für mich auf ein Machtverhältnis, bei der eine Seite das Recht hat Kritik zu üben, die andere nicht. Die mitunter enorme Entrüstung und Empörung darüber, die Partnerin würde einen verändern wollen, führt dann dazu, dass man sich zum Beispiel in der Paartherapie damit beschäftigt und nicht mehr mit dem kritisierten Verhalten. Sehr oft, so auch bei Peter, wird das Erleben von Inkompetenz über Rückzug abgewehrt. Die Paartherapeuten Hans Jellouschek (2006) sowie John Gottman (2000) gehen davon aus, dass es für gelingende Paarbeziehungen unerlässlich ist, dass man sich vom*von der Partner*in beeinflussen lässt und dies für Männer wesentlich schwieriger zu sein scheint.

- Die Rede vom Mann als drittem Kind verweist auf die Definitionsmacht der Frau in häuslichen und familiären Angelegenheiten; es wird die Funktionalisierung auf eine mütterliche Rolle beklagt und ein Machtverhältnis entworfen, in dem die Mutter-Frau den Sohn-Mann dominiert - eine dubiose Dominanz im Kontext patriarchaler Vermächtnisse, in denen Frauen ein schlechtes Gewissen haben, wenn sie sexuell nicht zu Diensten stehen und im Trennungsfall armutsgefährdet sind.

Der Leidensdruck von Petra und Peter ist nachvollziehbar hoch; meines Erachtens wird eine erfolgreiche Paartherapie im Sinn einer für beide befriedigendere und lebendigere Beziehung nicht ohne eine Reflexion sowie De- und Rekonstruktion ihrer Vorstellungen und Performanzen von Weiblichkeit und Männlichkeit auskommen. Ich wünsche mir für die beiden auch eine*n Paartherapeut*in, die*der aufgrund der eigenen Auseinandersetzung mit diesen Themen offen sein kann für die Gestaltung alternativer Möglichkeitsräume als Paar.

\section{Kybernetik II und gendersensible Systemiker*in- nen?}

Die systemische Familientherapie im deutschsprachigen Raum ist meiner Einschätzung nach eine psychotherapeutische Methode der Geschlechtsvergessenheit geblieben, die aufgrund der konservativ-männlichen Dominanz der Theoretiker im Schreiben und Lehren die zweite Frauenbewegung mit beharrlicher Resistenz „unbeschadet“ überstanden hat. Wiewohl im Lehrbuch der systemischen Therapie und Beratung (Jochen Schweitzer \& Arist von Schlippe) von $1996 \mathrm{zu}-$ mindest noch in dem Kapitel Gender-Sensitivity: Wie frauenfeindlich ist die systemische Therapie? eine wichtige Frage aufgeworfen wurde, so ist dieses reflexive Aufflackern in der 12 Jahre später erschienenen Neuauflage des Lehrbuchs (Schweitzer und von Schlippe 2008) völlig von der Bildfläche verschwunden. In dem von Tom Levold und Michael Wirsching 2014 herausgegebenen Systemische Therapie und Beratung 
- das große Lehrbuch finden sich vier Seiten zum Thema Geschlecht/Gender (Kirschenhofer 2014). Leider sehe ich auch keine integrative Reflexion im Sinn von Querschnittsmaterie Geschlecht, wodurch eigene Kapitel zum Thema obsolet würden.

Der Umstand, dass sich viele systemische Therapeut*innen der Illusion hingeben, sie wären „gendersensibel“, trägt zum Problem bei, weil sie glauben, sie müssten sich damit nicht mehr wirklich auseinandersetzen. Die theoretischen Grundlagen der systemischen Familientherapie nach der Wende von der Kybernetik I zu II ${ }^{5}$ werden sogar als Indiz eingeführt, wieso sich Systemiker*innen nicht speziell damit beschäftigten müssten: Erkenntnistheoretisch bedeutet Kybernetik II einen Abschied davon, ein System gezielt und mit ausgeklügelten Interventionen auf eine bestimmte Art und Weise verändern zu können ${ }^{6}$; stattdessen wurde der*die Beobachter*in/ Therapeut*in mit ihrer Mitverantwortung für Beobachtungen und ihre Rolle als Miterzeuger*in von Realitäten konzeptualisiert. Genau in dieser Konzeptualisierung läge und liegt auch die Chance der systemischen Therapie, sich qua ihrer Erkenntnistheorie mit ihrer Verantwortung bezüglich des Geschlechterverhältnisses auseinanderzusetzen. Nachdem eine solche Auseinandersetzung jedoch den derzeit dominierenden kapitalistischen Interessen ${ }^{7}$ widerspricht, so erscheinen stattdessen jedes Jahr zahlreiche systemische Werkzeug- und Fragetechniken-Bücher, deren Geschwätzigkeit in Sachen Machbarkeit an eine autoritärere Ära der Familientherapie erinnert.

Daneben gab und gibt es auch im deutschsprachigen Raum der systemischen Familientherapie eine feministische Tradition sich zu positionieren und zu publizieren, so zum Beispiel Irmgard Rücker-Emden-Jonasch und Andrea Ebbecke-Nohlen (1992), Rosmarie Welter-Enderlin (1996), Sabine Kirschenhofer und Verena Kuttenreiter (2006, 2010), zuletzt Angelika Grubner (2014) - manche von ihnen wurden inspiriert von U.S.-amerikanischen Familientherapeutinnen wie etwa Marianne Walters, Betty Carter, Peggy Papp und Olga Silverstein (1991) oder auch Rachel Hare-Mustin (1991, 1994).

\section{Von einer möglichen Annäherung an das Unmög- liche}

Wenn man sich für familientherapeutische und systemische Ansätze interessiert, die auch politische und soziale Kontexte integrieren, wird man eher im

\footnotetext{
5 Der Begriff Kybernetik (griech. kybernetes = Steuermann) stamm von dem Mathematiker Norbert Wiener und bedeutet Steuerungslehre.

${ }^{6}$ Für systemtheoretisches Denken im Sinn von Kybernetik II wurde der aus der Biologie stammende Begriff Autopoiese (griech. auto $=$ selbst und poiesie $=$ machen) einflussreich .

7 Siehe dazu Angelika Grubner (2017) Die Macht der Psychotherapie im Neoliberalismus.
}

englischsprachigen Raum fündig, wo feministische Familientherapie produktive und innovative Impulse biete ${ }^{8}$ und wo sich auch die narrative Therapie entwickelt hat. Ich werde hier einige Beispiele skizzieren und hoffe damit zu einer vertiefenden Auseinandersetzung mit der vorgestellten Literatur einzuladen:

Scheinkman und Fishbane (2006) etwa nutzen in der Paartherapie das von ihnen vorgestellte Modell des Vulnerabilitätskreislaufs: Die Verletzbarkeiten und Überlebensstrategien von Paaren werden verknüpft mit interaktionellen, soziokulturellen, intrapsychischen und intergenerationalen Bedeutungs- und Prozessebenen. Die Wirkmacht und Dekonstruktion von genderbezogenen Überzeugungen und Glaubenssätzen, die den Kreislauf destruktiv anheizen, ist Teil der therapeutischen Arbeit. So kann es möglich werden, dass z. B. ein Klient den Satz „Trau keiner Frau!“, den er aus seinem sozialen Umfeld übernommen hat, als Glaubenssatz aufgeben kann; er beginnt zu verstehen, wie negativ im Sinn eines Misstrauensgenerators sich dieser Satz auswirkt und auch nicht stimmt. Er kann erkennen, wie er die Partnerin mit seiner Eifersucht und Aggressivität einschüchtert, was zu einem Verhalten führt (Rückzug und manches aus Angst vor seinen Reaktionen nicht sagen), welches seine misstrauischen Gedanken bestätigt. In diesem Lernprozess kann er erfahren, dass sein Bemühen ihr mehr zu vertrauen dazu beiträgt, dass sie offener und selbstbewusster zeigen kann, was sie will und nicht will, was wiederum ihm ermöglicht, ihr mehr $\mathrm{zu}$ vertrauen.

Die narrative Therapie ${ }^{9}$ (vgl. Jill Freedman und Gene Combs 1996; Michael White und David Epston 1990; White 2007) hat sich über die Auseinandersetzung mit poststrukturalistischen Theorien und einer Rezeption von Michel Foucault unter anderem zum Ziel gesetzt, die Implikationen einer beobachterabhängigen Wirklichkeit (siehe weiter oben Stichwort Kybernetik II) ernst zu nehmen - inklusive einer Verantwortung für die eigenen Privilegien ${ }^{10}$.

Victoria Dickerson (2013), ebenfalls eine narrative Therapeutin, beschäftigt sich im Kontext der Paar-

\footnotetext{
8 Siehe zum Beispiel bei Teresa McDowell (2015) Applying Critical Social Theories to Family Therapy Practice.

9 Narrative Therapie gilt im deutschsprachigen Raum als eine Spezialisierung innerhalb der systemischen Therapie bzw. Arbeitsweise; die Gestaltung und Veränderung der eigenen Lebensgeschichte über das Entwerfen und Erzählen von alternativen Geschichten (Arbeit an bevorzugten Identitäten, Externalisierung von Problemen) ist Teil der Arbeit.

10 „We are educated, middle-aged embers of the professional class. As such, we are in positions of privilege in many contexts, including the therapy room. We do not want to reproduce, in therapy, the oppression many people have experienced at the hands of the dominant culture“ (Freedman und Combs 1996, S. 18). „We can reflect on the power relationships implicit in each possible discourse. We can seek new possibilities through selfeducation and through ongoing, regular deconstruction of our beliefs and practices“ (Freedman und Combs 1996, S. 58).
} 
therapie damit, wie man im Gewebe patriarchaler Machtbeziehungen arbeiten kann: In einer patriarchalen Kultur zu leben, bedeutet für Männer und Frauen, dass sie in ihrer geschlechtlichen Performanz, ihrem Repertoire $\mathrm{zu}$ denken, $\mathrm{zu}$ fühlen, $\mathrm{zu}$ handeln Dynamiken unterworfen sind, die für alle Geschlechter leidvoll und einschränkend sind. „Patriarchy intersects multiple sites in culture and moves through us; it captures our imaginations and enlists us as its agents“ (Dickerson 2013, S. 103). Es geht für Dickerson darum, wie wir im Dialog mit Klient*innen alternative Geschichten, Praktiken und Identitäten entwickeln können. Ein Weg führt über das Aufspüren des Abwesenden aber Impliziten, dem noch-nicht zum Ausdruck Gebrachten, das auch im Gespräch, im Raum, in der Luft liegt: Dass etwa eine Klage eine Sehnsucht beinhaltet, die es aufzuspüren gilt. Wenn ein Mann zum Beispiel beklagt, er könne nichts tun, wenn es der Frau schlecht geht, dann befindet er sich in einem Helfen-über-Tun Modus, der von der Partnerin vermutlich als nicht einfühlsam erfahren wird. Wenn dies übersetzt werden kann als Bedürfnis, Mitgefühl zu zeigen, dann verändert das möglicherweise die Bedeutung. Effekte des Patriarchats, wie Dickerson es nennen würde, werden auch darin sichtbar, dass sich Klient*innen mitunter nicht mit Zuschreibungen identifizieren wollen - bei einem Fallbeispiel zeigt sie, dass ihr Vorschlag, als Abwesendes aber Implizites „Mut“ zu konstruieren, daran scheitert, dass Mutigsein nicht in das Selbstbild der Klientin passt.

Abschließend werde ich ausgewählte Ergebnisse aus einem eigenen Forschungsprojekt zur Konstruktion von Geschlecht in Paartherapien (Kirschenhofer und Kuttenreiter 2006, 2010) skizzieren, woraus sich einige Empfehlungen für die Praxis ableiten lassen:

Wir analysierten ${ }^{11}$ drei videoaufgezeichnete Paartherapie-Sitzungen mit drei verschiedenen Paaren (samt jeweils unterschiedlichen Therapeut*innenPaaren). Dabei beschäftigten wir uns damit, wie Prozesse der (Re-)Konstruktion von männlichen und weiblichen Identitätsentwürfen gestaltet werden (können) und Machtkonstellationen repräsentiert und aktualisiert werden - und welche Position die Therapeut*innen dabei einnehmen. Judith Butler's Metapher der heterosexuellen Matrix aufgreifend strickt jede*r an dieser mit, über Art und Inhalt von Fragen, Aussagen, Reflexionen, über Blicke, Stimme, Tonfall, auch darüber, was (nicht) gehört wird. Die Ergebnisse waren durchaus ernüchternd dahingehend, dass unser Unbehagen (Vorannahme und Ausgangspunkt der Untersuchung) hinsichtlich der Stabilisierung leidvoller Dynamiken im paartherapeutischen Setting bestätigt wurde. Gerade diese unbehaglichen Erkenntnisse halten wir jedoch für fruchtbar, um etwa die in diesen

\footnotetext{
11 Wir arbeiteten im Rahmen unseres qualitativen sozialwissenschaftlichen Forschungsprojekts mit der Konversationsanalyse nach Deppermann (2001).
}

Videos vorgefundenen Konstellationen der heterosexuellen Matrix nicht zu reproduzieren.

- Die bereits weiter oben angesprochenen widersprüchlichen Erwartungen von Männern und Frauen an sich selbst und aneinander sind eine (auch gesellschaftliche) Realität, die nicht über das Herstellen von Eindeutigkeit aufgehoben werden kann: Die sich daraus entfaltenden Ambivalenzen mit Klient*innen auszuhalten kann zu einer wesentlichen Aufgabe der Therapeutin werden. Wenn Raum dafür geschaffen wird, erzeugt das eine Sicherheit, die eventuell zum Explorieren von neuen Wegen einlädt.

- Metaphern als Verstärker traditioneller Rollenbilder: Achtsamkeit im Umgang mit Metaphern und deren Passung in Bezug auf die von Klient*innen bevorzugten Geschlechts-Identitätsentwürfe ist gefragt. Wenn die Klientin die Metapher der im Turm auf Errettung durch den Prinzen wartenden Prinzessin einbringt, dann aber sagt, „so ist das aber nicht mehr“, dann widerstehen Sie als Therapeut*in der Versuchung, in traditionellen Märchenwelten an Identitäten herumzubasteln, die zurück in den Turm führen.

- Möglicherweise besteht für Therapeutinnen eine spezielle Verführung, zur Komplizin des „Veränderungsprojekts Mann“ zu werden. Also wenn sich zum Beispiel der Klient in eine Performanz als Kommunikationsmuffel hineinbewegt, kann es sein, dass er von der Therapeutin bearbeitet wird, seine kommunikativen Fähigkeiten auszubauen.

- Raum für affektive Kommunikation für beide Geschlechter ermöglichen: Nicht nur die Tränen des Mannes sind seltene und kostbare förderungswürdige Emotionen, auch die Frau soll diese im Paarsetting zeigen dürfen, ohne eines „mehr desselben“ verdächtigt und umgehend in konstruktivere Bahnen geführt zu werden.

- Autonomie und Macht: Die Person, die sich als der*die Autonomere präsentiert, bringt die andere, die davon spricht, die Beziehung unbedingt $\mathrm{zu}$ wollen, immer wieder in eine Position, noch mehr dafür zu tun. Erfahrungsgemäß zeigen sich Männer verbal häufiger als autonomer, wiewohl unsere körpersprachlichen Analysen spannende Diskrepanzen auf der nonverbalen Ebene zutage brachten. Therapeut*innen, die dieses Zusammenspiel zu wenig berücksichtigen, bestätigen und verstärken diese Konstellation.

Die Grenzen dessen, was im therapeutischen Kontext gespürt, gesehen, gedacht und gesagt werden kann, wird im Wesentlichen mitbestimmt durch das Ausmaß an Differenziertheit auf Seiten der*des Therapeut*in. Eine Anreicherung der Selbstreflexivität durch kontinuierliche eigene Auseinandersetzung mit dem Thema Geschlecht ist notwendig, um im Balanceakt eines Sowohl als auch beweglicher zu werden - sowohl die Berücksichtigung der Bedeutung von Unterschieden 
als auch ein Offenbleiben für die Einzigartigkeit von Menschen jenseits von Geschlecht.

Interessenkonflikt S. Kirschenhofer gibt an, dass kein Interessenkonflikt besteht.

Open Access Dieser Artikel wird unter der Creative Commons Namensnennung 4.0 International Lizenz (http:// creativecommons.org/licenses/by/4.0/deed.de) veröffentlicht, welche die Nutzung, Vervielfältigung, Bearbeitung, Verbreitung und Wiedergabe in jeglichem Medium und Format erlaubt, sofern Sie den/die ursprünglichen Autor(en) und die Quelle ordnungsgemäß nennen, einen Link zur Creative Commons Lizenz beifügen und angeben, ob Änderungen vorgenommen wurden.

\section{Literatur}

Butler, J. (1991). Das Unbehagen der Geschlechter. Frankfurt/M:Suhrkamp.

Deppermann, A. (2001). Gespräche analysieren. Eine Einführung (2. Aufl.). Reihe Qualitative Sozialforschung, Bd. 3. Opladen:Leske+Budrich

Dickerson, V. (2013). Patriarchy, power, and privilege: a narrative/poststructural view of work with couples. Family Process, 52(1), 102-114.

Freedman, J., \& Combs, G. (1996). Narrative therapy. The social construction of preferred realities. New York London: Norton.

Gottman, J. (2000). Die 7 Geheimnisse der glücklichen Ehe. Berlin: Ullstein.

Grubner, A. (2014). Geschlecht therapieren. Andere Erzählungen im Kontext narrativer systemischer Therapie. Heidelberg: Carl-Auer.

Grubner, A. (2017). Die Macht der Psychotherapie im Neoliberalismus. Eine Streitschrift. Wien: Mandelbaum.

Hare-Mustin, R. (1991). DieGeschlechterproblematikin derfamilientherapeutischen Theorie. In M. McGoldrick, C. M. Anderson \&F.Walsh (Hrsg.), Feministische Familientherapie in Theorie und Praxis(S.46-67). Freiburg im Breisgau: Lambertus.

Hare-Mustin, R. (1994). Diskurse im verspiegelten Raum Eine postmoderne Analyse der Therapie. Familiendynamik, 19(3), 205-232.

Jellouschek, H. (2006). Wie Liebe, Familie und Beruf zusammengehen. Partnerschaft heute. Freiburg im Breisgau: Herder.

Kirschenhofer, S. (2014). Geschlecht und Gender. In T. Levold \& M.Wirsching (Hrsg.), Systemische Therapie und Beratung - das große Lehrbuch (S.99-103). Heidelberg: Carl-Auer.
Kirschenhofer, S., \& Kuttenreiter, V. (2006). Die Wirksamkeit des Unsichtbaren. Konstruktion von Geschlecht in systemischen Paartherapien. Ergebnisse einer qualitativen Untersuchung. Wien:IEF.

Kirschenhofer, S., \& Kuttenreiter, V. (2010). Die Konstruktion von Geschlecht in Paartherapien. Ein Forschungsprojekt. In A. Brandl-Nebehay \& J. Hinsch (Hrsg.), Paartherapie und Identität(S. 80-108). Heidelberg: Carl-Auer.

Lerner, G. (1986). The creation of patriarchy. New York: Oxford University Press.

McDowell, T. (2015). Applying critical social theories to family therapy practice. Cham Heidelberg New York Dordrecht London:AFTASpringer.

McGeorge, C., \&Carlson, S. T. (2011). Deconstructingheterosexism: Becoming an LGB affirmative heterosexual couple and family therapist. Journal of Marital and Family therapy, 37(1), 14-26.

Meuser, M. (2009). Hegemoniale Männlichkeit - Überlegungen zur Leitkategorie der Men's Studies. In B. Aulenbacher, et al. (Hrsg.), FrauenMännerGeschlechterforschung. State of the Art (S. 160-174). Münster: Westfälisches Dampfboot.

Ogette, T. (2017). exit RACISM rassismuskritisch denken lernen. Münster: Unrast.

Rücker-Emden-Jonasch, I., \& Ebbecke-Nohlen, A. (Hrsg.). (1992). Balanceakte. Familientherapie und Geschlechterrollen. Heidelberg: Carl-Auer.

Scheinkman, M., \& Fishbane, M. (2006). Der Vulnerabilitätskreislauf:Der UmgangmitSackgassen in der Paartherapie. Familiendynamik, 31, 152-179.

Schweitzer, J., \& Von Schlippe, A. (2008). Lehrbuch der systemischen Therapie und Beratung I. Das Grundlagenwissen. Göttingen:Vandenhoeck\&Ruprecht.

Walters, M., Carter, B., Papp, P., \& Silverstein, O. (1991). Unsichtbare Schlingen. Die Bedeutung der Geschlechterrollen in der Familientherapie. Eine feministische Perspektive. Stuttgart: Klett-Cotta.

Welter-Enderlin, R. (1996). Deine Liebe ist nicht meine Liebe. Partnerprobleme und Lösungsmodelle aus systemischer Sicht. Freiburgim Breisgau:Herder.

White, M. (2007). Maps of narrative practice. New York/ London: Norton.

White, M., \& Epston, D. (1990). Die Zähmung der Monster: Der narrative Ansatz in der Familientherapie. Heidelberg: CarlAuer.

Hinweis des Verlags Der Verlag bleibt in Hinblick auf geografische Zuordnungen und Gebietsbezeichnungen in veröffentlichten Karten und Institutsadressen neutral. 